\title{
HYPONORMALITY OF TOEPLITZ OPERATORS
}

\author{
CARL C. COWEN
}

(Communicated by John B. Conway)

\begin{abstract}
For $\varphi$ in $L^{\infty}(\partial \mathbf{D})$, let $\varphi=f+\bar{g}$ where $f$ and $g$ are in $H^{2}$. In this note, it is shown that the Toeplitz operator $T_{\varphi}$ is hyponormal if and only if $g=c+T_{\bar{h}} f$ for some constant $c$ and some function $h$ in $H^{\infty}(\mathrm{D})$ with $\|h\|_{\infty} \leq 1$.
\end{abstract}

For $\varphi$ in $L^{\infty}(\partial \mathrm{D})$, the Toeplitz operator $T_{\varphi}$ is the operator on $H^{2}$ of the unit disk D given by $T_{\varphi} u=P \varphi u$ where $P$ is the orthogonal projection of $L^{2}(\partial \mathrm{D})$ onto $H^{2}$. An operator $A$ is called hypnormal if its self-commutator $A^{*} A-A A^{*}$ is positive. The goal of this paper is to characterize hyponormal Toeplitz operators.

Brown and Halmos began the systematic study of the algebraic properties of Toeplitz operators and showed, [3, p. 98], that $T_{\varphi}$ is normal if and only if $\varphi=\alpha+\beta \rho$ where $\alpha$ and $\beta$ are complex numbers and $\rho$ is a real valued function in $L^{\infty}$. There are many results concerning hyponormality of Toeplitz operators in the literature and properties of hyponormal Toeplitz operators have played an important role in work on Halmos's Problem 5, [7], "Is every subnormal Toeplitz operator either normal or analytic?" but a characterization has been lacking. (For references, see the bibliography; [6] surveys much of the literature.)

THEOREM 1. If $\varphi$ is in $L^{\infty}(\partial \mathbf{D})$, where $\varphi=f+\bar{g}$ for $f$ and $g$ in $H^{2}$, then $T_{\varphi}$ is hyponormal if and only if

$$
g=c+T_{\bar{h}} f
$$

for some constant $c$ and some function $h$ in $H^{\infty}(\mathbf{D})$ with $\|h\|_{\infty} \leq 1$.

The basis of the proof is a dilation theorem; we will use the notation and formulation of Sarason [13, Theorem 1]. The unilateral (forward) shift on $H^{2}$ will be denoted by $U$. Moreover, the proof uses standard results about Hankel operators, for example, see [12]. For $\psi$ in $L^{\infty}$, the Hankel operator $H_{\psi}$ is the operator on $H^{2}$ given by

$$
H_{\psi} u=J(I-P)(\psi u)
$$

where $J$ is the unitary operator from $H^{2 \perp}$ onto $H^{2}$

$$
J\left(e^{-i n \theta}\right)=e^{i(n-1) \theta} .
$$

Denoting by $v^{*}$ the function $v^{*}\left(e^{i \theta}\right)=\overline{v\left(e^{-i \theta}\right)}$, another way to put this is that $H_{\psi}$ is the operator on $H^{2}$ defined by

$$
\langle z u v, \bar{\psi}\rangle=\left\langle H_{\psi} u, v^{*}\right\rangle, \quad \text { for all } v \in H^{\infty} .
$$

Received by the editors June 3, 1987. Presented January 7, 1988 to the Session on Operator Theory at the 94th Annual Meeting of the American Mathematical Society.

1980 Mathematics Subject Classification (1985 Revision). Primary 47B35, 47B20.

Key words and phrases. Toeplitz operator, subnormal operator.

Supported in part by National Science Foundation Grant DMS 87-10006. 
Necessary facts about Hankel operators include

- $H_{\psi_{1}}=H_{\psi_{2}}$ if and only if $(I-P) \psi_{1}=(I-P) \psi_{2}$.

- $\left\|H_{\psi}\right\|=\inf \left\{\|\varphi\|_{\infty}:(I-P) \psi=(I-P) \varphi\right\}$.

- $H_{\psi}^{*}=H_{\psi^{*}}$.

- Either $H_{\psi}$ is one-to-one or $\operatorname{ker}\left(H_{\psi}\right)=\chi H^{2}$ where $\chi$ is an inner function. The closure of the range of $H_{\psi}$ is $H^{2}$ in the former case and $\left(\chi^{*} H^{2}\right)^{\perp}$ in the latter.

- $H_{\psi} U=U^{*} H_{\psi}$.

ProOF. Let $\varphi=f+\bar{g}$ where $f$ and $g$ are in $H^{2}$.

The first step of the proof is one of the equivalences of Proposition 11 of [6]. For every polyomial $p$ in $H^{2}$,

$$
\begin{aligned}
\left\langle\left(T_{\varphi}^{*} T_{\varphi}-T_{\varphi} T_{\varphi}^{*}\right)(p), p\right\rangle & =\left\langle T_{\varphi} p, T_{\varphi} p\right\rangle-\left\langle T_{\varphi}^{*} p, T_{\varphi}^{*} p\right\rangle \\
& =\langle f p+P \bar{g} p, f p+P \bar{g} p\rangle-\langle P \bar{f} p+g p, P \bar{f} p+g p\rangle \\
& =\langle\bar{f} p, \bar{f} p\rangle-\langle P \bar{f} p, P \bar{f} p\rangle-\langle\bar{g} p, \bar{g} p\rangle+\langle P \bar{g} p, P \bar{g} p\rangle \\
& =\langle\bar{f} p,(I-P) \bar{f} p\rangle-\langle\bar{g} p,(I-P) \bar{g} p\rangle \\
& =\langle(I-P) \bar{f} p,(I-P) \bar{f} p\rangle-\langle(I-P) \bar{g} p,(I-P) \bar{g} p\rangle \\
& =\left\|H_{\bar{f}} p\right\|^{2}-\left\|H_{\bar{g}} p\right\|^{2} .
\end{aligned}
$$

Since the polynomials are dense in $\mathrm{H}^{2}$ and since the Hankel and Toeplitz operators involved are bounded, we see that $T_{\varphi}$ is hyponormal if and only if for all $u$ in $H^{2}$,

$$
\left\|H_{\bar{g}} u\right\| \leq\left\|H_{\bar{f}} u\right\| .
$$

Let $K$ denote the closure of the range of $H_{\bar{f}}$, and let $S$ denote the compression of $U$ to $K$. Since $K$ is invariant for $U^{*}$, the operator $S^{*}$ is the restriction of $U^{*}$ to $K$. by

Suppose first that $T_{\varphi}$ is hyponormal. Define an operator $A$ on the range of $H_{\bar{f}}$

$$
A\left(H_{\bar{f}} u\right)=H_{\bar{g}} u .
$$

If $H_{\bar{f}} u_{1}=H_{\bar{f}} u_{2}$, so that $H_{\bar{f}}\left(u_{1}-u_{2}\right)=0$, then the inequality (2) implies that $H_{\bar{g}}\left(u_{1}-u_{2}\right)=0$ too and it follows that $A$ is well defined. Moreover, inequality (2) implies $\|A\| \leq 1$ so $A$ has an extension to $K$, which will also be denoted $A$, with the same norm.

Now by the intertwining formula for Hankel operators and the fact that $K$ is invariant for $U^{*}$, we have

$$
H_{\bar{g}} U=A H_{\bar{f}} U=A U^{*} H_{\bar{f}}=A S^{*} H_{\bar{f}}
$$

and also

$$
H_{\bar{g}} U=U^{*} H_{\bar{g}}=U^{*} A H_{\bar{f}}=S^{*} A H_{\bar{f}} .
$$

Since the range of $H_{\bar{f}}$ is dense in $K$, we find that $A S^{*}=S^{*} A$ on $K$, or taking adjoints, that

$$
S A^{*}=A^{*} S \text {. }
$$

By [13, Theorem 1] (or by the usual theory of the unilateral shift if $K=H^{2}$ ), there is a function $k$ in $H^{\infty}(\mathbf{D})$ with $\|k\|_{\infty}=\left\|A^{*}\right\|=\|A\|$ such that $A^{*}$ is the 
compression to $K$ of $T_{k}$. Since $K$ is invariant for $T_{k}^{*}=T_{\bar{k}}$, this means that $A$ is the restriction of $T_{\bar{k}}$ to $K$ and

$$
H_{\bar{g}}=T_{\bar{k}} H_{\bar{f}} \text {. }
$$

Conversely, if equation (3) holds for some $k$ in $H^{\infty}$ (D) with $\|k\|_{\infty} \leq 1$, then clearly inequality (2) holds for all $u$, and $T_{\varphi}$ is hyponormal.

The proof will be completed by analyzing the relationship given by equation (3). Using the formulation (1), equation (3) holds if and only if for all $H^{\infty}$ functions $u$, $v$,

$$
\begin{aligned}
\langle z u v, g\rangle & =\left\langle H_{\bar{g}} u, v^{*}\right\rangle=\left\langle T_{\bar{k}} H_{\bar{f}} u, v^{*}\right\rangle=\left\langle H_{\bar{f}} u, k v^{*}\right\rangle \\
& =\left\langle z u k^{*} v, f\right\rangle=\left\langle z u v, \overline{k^{*}} f\right\rangle=\left\langle z u v, T_{\overline{k^{*}}} f\right\rangle .
\end{aligned}
$$

Since the closed span of $\left\{z u v: u, v \in H^{\infty}\right\}$ is $z H^{2}$ this means that equation (3) holds if and only if $g=c+T_{\bar{h}} f$ for $h=k^{*}$. (Note that $\|k\|_{\infty}=\left\|k^{*}\right\|_{\infty}$.)

In the cases for which $T_{\varphi}$ is normal, $h$ is a constant of modulus 1 and in the cases for which $T_{\varphi}$ is known to be subnormal but not normal, $h$ is a constant of modulus less than 1 .

It is of some interest to investigate the uniqueness of the functions $h$ that relate $f$ and $g$. Suppose $h_{1}$ and $h_{2}$ are in $H^{\infty}$ and $c_{1}+T_{\overline{h_{1}}} f=g=c_{2}+T_{\overline{h_{2}}} f$. This is possible if and only if

$$
T_{\bar{z}} T_{\overline{h_{1}}} f=T_{\bar{z}} T_{\overline{h_{2}}} f
$$

that is, if and only if $T_{\overline{z h_{1}-z h_{2}}} f=0$. Thus, $f$ must be in $\left(z \chi H^{2}\right)^{\perp}$ where $\chi$ is the inner factor of $h_{1}-h_{2}$. If $f$ is not in any such subspace, the corresponding function $h$ must be unique for every $g$. On the other hand, if $\chi$ is an inner function such that $f$ is in $\left(z \chi H^{2}\right)^{\perp}$ and $c_{1}+T_{\overline{h_{1}}} f=g$, then for any $h_{3}$ in $H^{\infty}$ and $h_{2}=h_{1}+z \chi h_{3}$, it follows that $g=c_{2}+T_{\overline{h_{2}}} f$ for some constant $c_{2}$.

In [6], the author made the following generalization of the set of $g$ in $H^{2}$ for which $T_{f+\bar{g}}$ is hyponormal.

Definition. Let $\mathscr{H}=\left\{v \in H^{\infty}: v(0)=0\right.$ and $\left.\|v\|_{2} \leq 1\right\}$. For $f$ in $H^{2}$, let $G_{f}$ denote the set of $g$ in $H^{2}$ such that for every $u$ in $H^{2}$,

$$
\sup _{v_{0} \in \mathscr{Z}}\left|\left\langle u v_{0}, g\right\rangle\right| \leq \sup _{v_{0} \in \mathscr{H}}\left|\left\langle u v_{0}, f\right\rangle\right| \text {. }
$$

To see how this definition is relevant to our work, note that if $f$ is in $H^{\infty}$ and $u$ is in $H^{2}$, then by equation (1),

$$
\sup _{v_{0} \in \mathscr{R}}\left|\left\langle u v_{0}, f\right\rangle\right|=\left\|H_{\bar{f}} u\right\| .
$$

Thus, when $f$ and $g$ are bounded analytic, $T_{f+\bar{g}}$ is hyponormal if and only if $g$ is in $G_{f}$.

For $f$ in $H^{2}$, not necessarily the analytic part of a function in $L^{\infty}$, if we regard $H_{\bar{f}}$ as a bounded operator from $H^{\infty}$ into $H^{2}$, then we may proceed exactly as above to prove the following theorem.

THEOREM 2. If $f$ and $g$ are in $H^{2}$, then $g$ is in $G_{f}$ if and only if $g=c+T_{\bar{h}} f$, for some constant $c$ and some function $h$ in $H^{\infty}(\mathbf{D})$ with $\|h\|_{\infty} \leq 1$.

We can now easily answer Question 1 of [6]. 
COROLlaRY 3. For $f$ in $\mathrm{H}^{2}$, the following hold.

(1) $f$ is in $G_{f}$.

(2) If $g$ is in $G_{f}$, then $g+\lambda$ is in $G_{f}$ for all complex numbers $\lambda$.

(3) $G_{f}$ is balanced and convex; that is, if $g_{1}$ and $g_{2}$ are in $G_{f}$ and $\left|s_{1}\right|+\left|s_{2}\right| \leq 1$, then $s_{1} g_{1}+s_{2} g_{2}$ is also in $G_{f}$.

(4) $G_{f}$ is weakly closed.

(5) $T_{\bar{\chi}} G_{f} \subset G_{f}$ for every inner function $\chi$.

Conversely, if $G$ is a set that satisfies properties (1) to (5), then $G \supset G_{f}$.

PROOF. That $G_{f}$ has the indicated properties is Theorem 12 of [6].

To prove the converse statement, note that $f$ is in $G$ and by (3), (4), and (5), $G$ contains $T_{\bar{h}} f$ whenever $h$ is in the weakly closed convex hull of the set of inner functions. By a theorem of Marshall [11, Corollary, p. 496], the norm closed convex hull of the Blaschke products in $H^{\infty}$ is the unit ball of $H^{\infty}$. Property (2) and Theorem 2 now imply the desired inclusion.

\section{REFERENCES}

1. M. B. Abrahamse, Subnormal Toeplitz operators and functions of bounded type, Duke Math. J. 43 (1976), 597-604.

2. I. Amemiya, T. Ito, and T. K. Wong, On quasinormal Toeplitz operators, Proc. Amer. Math. Soc. 50 (1975), 254-258.

3. A. Brown and P. R. Halmos, Algebraic properties of Toeplitz operators, J. Reine Angew. Math. 213 (1963-64), 89-102.

4. C. C. Cowen and J. J. Long, Some subnormal Toeplitz operators, J. Reine Angew. Math. 351 (1984), 216-220.

5. C. C. Cowen, More subnormal Toeplitz operators, J. Reine Angew. Math. 367 (1986), 215-219.

6. - Hyponormal and subnormal Toeplitz operators, Surveys of Recent Results in Operator Theory, vol. 1 (J. B. Conway and B. B. Morrel, eds.) (to appear).

7. P. R. Halmos, Ten problems in Hilbert space, Bull. Amer. Math. Soc. 76 (1970), 887-933.

8. __ Ten years in Hilbert space, J. Integral Equations and Operator Theory 2 (1979), 529 564.

9. T. Ito and T. K. Wong, Subnormality and quasinormality of Toeplitz operators, Proc. Amer. Math. Soc. 34 (1972), 157-164.

10. J. J. Long, Hyponormal Toeplitz operators and weighted shifts, Thesis, Michigan State Univ., 1984.

11. D. E. Marshall, Blaschke products generate $H^{\infty}$, Bull. Amer. Math. Soc. 82 (1976), 494-496.

12. S. C. Power, Hankel operators on Hilbert space, Pitman, Boston, Mass., 1982.

13. D. Sarason, Generalized interpolation in $H^{\infty}$, Trans. Amer. Math. Soc. 127 (1967), 179-203.

14. Sun Shunhua, Bergman shift is not unitarily equivalent to a Toeplitz operator, Kexue Tongbao 28 (1983), 1027-1030.

15. __, On Toeplitz operators in the $\Theta$-class, Scientia Sinica (Series A) 28 (1985), 235-241.

Department of Mathematics, PuRdue University, West LAFAyette, Indiana 47907 